\title{
Intravascular Lymphoma as an Uncommon Cause of Anasarca
}

\author{
Eleni Mylona ${ }^{1}$, Styliani Golfinopoulou ${ }^{1}$, Pelagia Sfakianaki ${ }^{1},{ }^{1}$ George Kyriakopoulos ${ }^{2}$, Ioannis Tsonis ${ }^{3}$, \\ Theofanis Apostolou ${ }^{4}$, Christina Vourlakou ${ }^{2}$, Athanasios Skoutelis ${ }^{1}$
}

${ }_{1}^{1}$ Fifth Department of Medicine, Evangelismos General Hospital, Athens, Greece; ${ }^{2}$ Pathology Department, Evangelismos General Hospital, Athens, Greece; ${ }^{3}$ Hematology Department, Evangelismos General Hospital, Athens, Greece; ${ }^{4}$ Nephrology Department, Evangelismos General Hospital, Athens, Greece

Received: 09/03/2016

Accepted: 01/06/2016

Published: 29/06/2016

How to cite this article: Mylona E, Golfinopoulou S, Sfakianaki P, Kyriakopoulos G, Tsonis I, Apostolou T, Vourlakou C, Skoutelis A. Intravascular lymphoma as an uncommon cause of anasarca. EJCRIM 2016;3:doi:10.12890/2016 000424

Conflicts of Interests: The Authors declare that there are no competing interests.

This article is licensed under a Commons Attribution Non-Commercial 4.0 License

\section{ABSTRACT}

Objectives: To report a case of intravascular lymphoma (IVL) in a Caucasian patient who presented with anasarca as his sole clinical sign. Material and Methods: A man presented with anasarca-type oedema and fatigue. After excluding heart failure, hepatic cirrhosis, nephrotic syndrome, hypothyroidism, AL-amyloidosis and adverse drug reaction which can all cause oedema, we turned our attention to capillary permeability disorders.

Results: Closer review of the bone marrow aspirate demonstrated haemophagocytic histiocytosis, while core, renal and duodenal biopsies showed a B-cell IVL.

Conclusion: The differential diagnosis of anasarca, a relatively common clinical sign, should include IVL although the diagnosis may still be challenging.

\section{LEARNING POINTS}

- Anasarca-type oedema is an unusual initial presentation of intravascular lymphoma (IVL) and is normally attributed to capillary permeability disorders.

- Two clinical forms of IVL have been recognized: a Western form and an Asian variant which is characterized by haemophagocytosis.

- Patients of Caucasian origin who have the clinical features of the Asian variant of IVL make the diagnosis of this condition even more challenging.

\section{KEYWORDS}

Anasarca oedema, haemophagocytosis, intravascular lymphoma.

\section{INTRODUCTION}

Oedema is an accumulation of fluid in the intercellular tissue, which when generalized is also called anasarca. Anasarca occurs when systemic conditions disrupt the equilibrium between the hydrostatic and the oncotic pressure gradients across capillaries, thus leading to increased capillary hydrostatic pressure, decreased plasma oncotic pressure, increased capillary permeability and/or lymphatic obstruction ${ }^{[1]}$. Intravascular large B-cell lymphoma is a rare type of extranodal large B-cell lymphoma characterized by the selective growth of neoplastic cells within the lumina of capillaries ${ }^{[2]}$. This behaviour means any organ can be involved. Consequently, the clinical manifestations of IVL are variable as they are related to the infiltrated organ, making diagnosis challenging ${ }^{[3]}$. Anasarca-type oedema is an unusual initial presentation, reported in less than $5 \%$ of patients in one series.It may be caused by increased capillary permeability due to endothelial damage as a result 
of the IVL or by vascular obstruction because of aggregation of lymphoma cells within the small vessels ${ }^{[4]}$.

We report the case of a Caucasian man with IVL who presented with anasarca as his sole clinical sign.

\section{CASE REPORT}

A 70-year-old Caucasian male presented to the emergency department of our hospital with a 6-month history of oedema, dyspnoea and fatigue. The patient denied fever, orthopnoea or weight loss.

His past medical history was notable for hypertension and diabetes as well as spleenectomy following an accident 25 years previously. His medications included vildagliptin, gliclazide and diltiazem.

On physical examination, the patient was afebrile. His blood pressure was 150/90 mmHg, pulse was 80 bpm and respiratory rate was 24 breaths/min. His oxygen saturation was $88 \%$ while breathing ambient air, but $93 \%$ with a nasal cannula at a flow rate of $2 \mathrm{l} / \mathrm{min}$. The rest of the examination was remarkable for anasarca oedema without jugular distention. The lungs were clear on auscultation and the abdomen was soft and without tenderness. There was no lymphadenopathy or rash and the neurological examination was normal.

Laboratory studies revealed the following: haemoglobin, $13.7 \mathrm{~g} / \mathrm{dl}$; white blood cell count, $7.1 \times 103 / \mu \mathrm{l}$; platelet count, $98 \times 103 / \mu \mathrm{l}$; serum urea nitrogen, $90 \mathrm{mg} / \mathrm{dl}$; and creatinine, $1.88 \mathrm{mg} / \mathrm{dl}$ (which had increased from a baseline of $0.78 \mathrm{mg} / \mathrm{dl}$ measured 2 months previously). Liver function tests were normal, while lactate dehydrogenase (LDH) was $619 \mathrm{IU} / \mathrm{I}$. Serum albumin was $3.2 \mathrm{~g} / \mathrm{dl}$. C-reactive protein (CRP) was 5.1 $\mathrm{mg} / \mathrm{dl}$ (normal: $<0.5 \mathrm{mg} / \mathrm{dl}$ ) and the erythrocyte sedimentation rate (ESR) was $58 \mathrm{~mm} / \mathrm{h}$. The lipid panel, protein electrophoresis as well as thyroid hormones and the international normalized ratio (INR) were all normal. Ferritin was $199 \mathrm{ng} / \mathrm{ml}$ and the $\beta 2$-microglobulin level was $4.697 \mu \mathrm{g} / \mathrm{l}$. Blood cultures were also obtained despite lack of fever, but demonstrated no growth. Urinalysis identified no casts, red blood cells or crystals but only trace proteins, corresponding to a $24 \mathrm{~h}$ urinary protein value of $340 \mathrm{mg}$.

EKG was normal. Chest radiograph only revealed emphysema. Ultrasonography of the abdomen and computed tomography (CT) scan of the abdomen demonstrated a small amount of ascitic fluid, and three nodules at the anatomic site of the spleen, which after contrast infusion were characterized as a regenerated spleen. Because of tachypnoea and hypoxaemia, the patient underwent CT pulmonary angiography (CTPA) which was negative for pulmonary embolism. CTPA only demonstrated small bilateral pleural effusions and emphysema. The ascitic and pleural fluids were found to be transudates. An echocardiogram demonstrated normal right and left ventricular function with an ejection fraction of $>60 \%$. An upper endoscopy was also performed. No oesophageal varices were found and blind biopsies from the stomach and duodenum were obtained.

Our focus then shifted to haematological malignancy because of the increased LDH and $\beta 2$-microglobulin as well as thrombocytopenia. Bone marrow aspiration demonstrated intense haemophagocytic histiocytosis, although the criteria for haemophagocytic syndrome were not met. Core biopsy showed monoclonal B lymphocytes with the characteristics of intravascular lymphoma. In the meantime, because of the mild renal failure, a renal biopsy had been obtained which, together with the duodenal biopsy, further confirmed the diagnosis of intravascular lymphoma (Fig. 1).

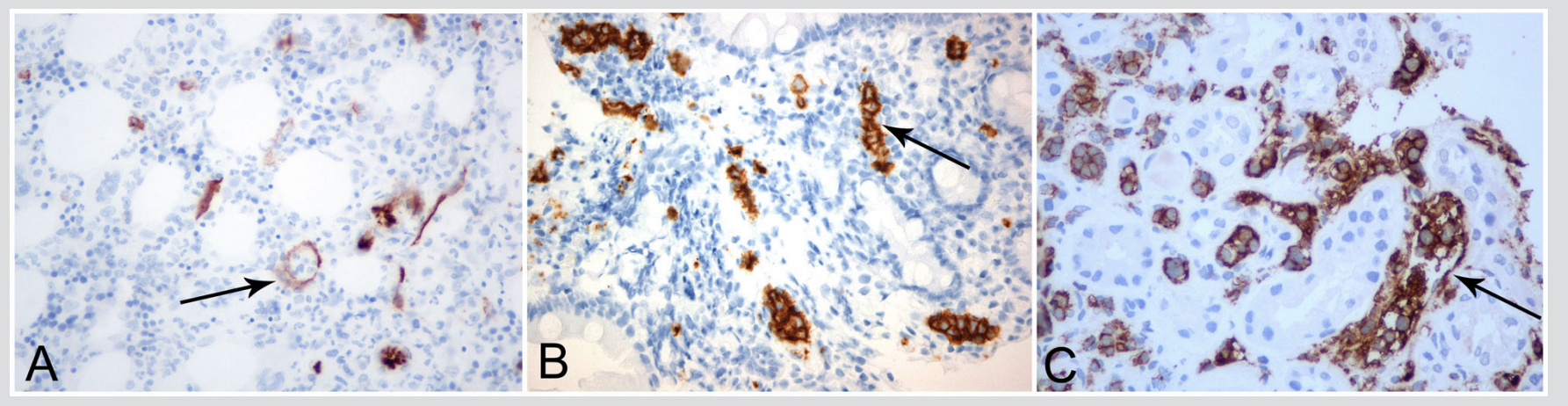

Figure 1: Representative photographs of immunohistochemical staining with diaminobenzidine (A,B 200x magnification, C 400x). (A) Arrow indicates intravascular lymphocytes with enlarged nuclei in bone marrow biopsy sample stained with CD34 antibody. (B,C) Arrows indicate aggregates of intravascular lymphocytes stained positively with CD2O antibody in duodenal and renal biopsy samples, respectively. 
At this time, the patient presented low grade fever and became increasingly dyspnoeic and hypoxaemic requiring more supplemental oxygen. A new chest CT scan revealed infiltrates in both lungs and the patient was administered meropenem but without response. Bronchoscopy revealed friable mucous membranes which bled easily, while cultures of bronchial secretions did not reveal any pathogens. Thus, dissemination of IVL in the lungs was considered probable and treatment with R-CHOP (rituximab, vincristine, doxorubicin, cyclophosphamide and prednisolone) chemotherapy was initiated. The patient died from multiple organ failure 1 week after the first cycle of chemotherapy.

\section{DISCUSSION}

The patient described here presented with anasarca-type oedema and fatigue as his only complaints. The initial differential diagnosis for his anasarca included heart failure, renal failure/nephrotic syndrome, liver cirrhosis, hypothyroidism, systemic amyloidosis, adverse drug reaction and systemic capillary leak syndrome.

Since congestive or right-sided heart failure is the most common cause of anasarca, our first concern was to check cardiac function. However, the patient's history (no orthopnoea or paroxysmal dyspnoea), physical examination (no jugular distension) and imaging examinations (chest radiograph, echocardiogram, CTPA) ultimately excluded cardiac dysfunction as a cause of anasarca. His medication did not include any drugs implicated in anasarca. The initial blood work ruled out hypothyroidism as well as hypoalbuminaemia and therefore any other protein wasting condition such as nephrotic syndrome or protein wasting enteropathy.

The patient's renal failure (estimated glomerular filtration rate $\sim 38 \mathrm{ml} / \mathrm{min} / 1.73 \mathrm{~m}^{2}$ ) was not bad enough to definitely indicate anasarca, although in conjunction with the elevated ESR and CRP it did raise concern about an underlying systemic inflammatory or neoplastic process. However, a normal echocardiogram and normal protein electrophoresis as well as the lack of hypoalbuminaemia made systemic amyloidosis less likely.

A striking feature of the patient's initial blood work was thrombocytopenia which combined with ascites raised concern about liver failure. However, the albumin gradient of the ascitic fluid, the lack of findings of hepatic synthetic dysfunction (normal albumin and INR) as well as a lack of varices made a hepatic cause of the anasarca a remote possibility. Furthermore, the CT scan excluded inferior vena cava or iliac vein thrombosis. However, while the patient's past history included spleenectomy after a car accident, the CT scan showed nodules at the anatomic site of the spleen which acted as a spleen after contrast infusion. This made us consider that either the patient had an accessory spleen or, less likely, that a piece of the spleen had broken off with the trauma and, under possible immunological pressure, grew and functioned as a spleen.

Since the work-up so far had excluded hydrostatic and colloid osmotic causes of oedema, we turned our attention to capillary permeability disorders. The patient did not fulfil criteria for systemic capillary leak syndrome which is characterized by unexplained paroxysmal capillary hyperpermeability resulting in hypotension, haemoconcentration and hypoalbuminaemia ${ }^{[5]}$. Therefore and in view of the increased LDH and $\beta 2$-microglobulin as well as the thrombocytopenia and regenerated spleen, which was considered equivalent to splenomegaly, we considered lymphoma as a potential diagnosis.

Two clinical forms of IVL have been recognized in the literature: a Western form, displaying a relatively high frequency of central nervous system and skin involvement ${ }^{[4,6]}$ and an Asian variant, mostly reported in the Japanese literature, which is associated with haemophagocytosis ${ }^{[6,7]}$. Our patient's IVL had the characteristics of the Asian variant despite his Caucasian origin.

In conclusion, this case highlights that in the differential diagnosis of anasarca, a relatively common clinical sign, IVL should be included although it is an extremely rare disease with variable manifestations making its diagnosis very difficult.

\section{REFERENCES}

1. Guyton AC, Hall JE. The body fluid compartments: extracellular and intracellular fluids; interstitial fluid and edema. In: Guyton AC, Hall JE, editors. Textbook of Medical Physiology. Philadelphia: WB Saunders, 1991:274-285.

2. Nakamura S, Ponzoni M, Campo E. Intravascular large B-cell lymphoma. In: Swerdlow SH, Campo E, Harris NL, et al, editors. WHO Classification of Tumours of Haematopoietic and Lymphoid Tissues. Lyon, France: IARC Press, 2008:252-253.

3. Shimada K, Kinoshita T, Naoe T, Nakamura S. Presentation and management of intravascular large B-cell lymphoma. Lancet 2009;10:895-902.

4. Ferreri AJ, Campo E, Seymour JF, Willemze R, Illariucci F, Ambrosetti A, et al. Intravascular lymphoma: clinical presentation, natural history, management and prognostic factors in a series of 38 cases, with special emphasis on the "cutaneous variant". Br J Haematol 2004;127:173-183.

5. Bonadies N, Baud P, Peter H-J, Buergi U, Mueller BU. A case report of Clarkson's disease: if you don't know it, you'll miss it. Eur J Intern Medicine 2006;17:363-365.

6. Ponzoni M, Ferreri JM. Intravascular lymphoma: a neoplasm of "homeless" lymphocytes? Hematol Oncol 2006;24:105-112.

7. Murase T, Nakamura S, Kawauchi K, Matsuraki H, Sakai C, Inaba T, et al. An Asian variant of intravascular large B-cell lymphoma: clinical, pathological and cytogenetic approaches to diffuse large B-cell lymphoma associated with haemophagocytic syndrome. Br J Haematol 2000;111:826-834. 